\title{
Effects of Teacher Support on College Students' Math Achievement: The Mediating Effects of Basic Psychological Needs and Extrinsic Motivation
}

\author{
Hongye Wu \\ Huizhou University, Huizhou, China \\ Ke Wang \\ South China Normal University, Guangzhou, China \\ Haiqing Wang \\ Huizhou University, Huizhou, China
}

This paper investigated the effects of teacher support on college students' math achievement. The sample included 1,176 college students from a local university in Guangdong Province, China. Guided by self-determination theory, college students' basic psychological needs and extrinsic motivation were measured with three metrics including a basic psychological need scale, a learning motivation scale, and a teacher support scale. The results indicated that: (1) Liberal arts and science students had significant differences in their perceptions about teacher support and relationship support; (2) Teacher support had a positive predictive effect on college students' math achievement; (3) Basic psychological needs and extrinsic motivation played a mediating role between teacher support and college students' math achievement; and (4) The mediating effect of extrinsic motivation on students' math achievement was greater than students' basic psychological needs.

Keywords: teacher support, basic psychological needs, extrinsic motivation, college students, mathematics achievement

Research indicates that there are many factors affecting students' math achievement, including personal intelligence and non-intelligence, teachers, school factors, and family factors (Qiu \& Gao, 1999; Xiao, Zhang, \& Shi, 2015). Among those factors, teacher support prevails as an important environmental factor that significantly affects college student math achievement (Lu, 2010). Scholars at home and abroad have done a lot of research on the relationship between teacher support perceived by students and students' math achievement. In order to understand the internal mechanism, some scholars have conducted empirical research on the relationship among teacher support, basic psychological needs, internal 
motivation and college students' math achievement (Zhang, Fu, \& Liu, 2018). However, there is less research on the intermediary effect of teacher support on academic performance through the satisfaction of basic psychological needs and extrinsic motivation, and on the effect mechanism of teacher support perceived by local college students on their math performance through Structural Equation Modeling (SEM). Therefore, based on self-determination theory and the existing research, this study explored the mechanism of teacher support affecting college students' math achievement, with students' basic psychological needs and extrinsic motivation as the variables for investigating the effects of the mediating roles of basic psychological needs and extrinsic motivation.

\section{Teacher Support}

There are a number of teacher supports beneficial for students. Teacher support in this study refers to the competence support, autonomous support and relationship support provided by teachers to students in the classroom. Competence support includes feedback, some criticism, establishing expectations and instrumental assistance to students. Autonomous support includes adopting students' views, fully respecting students, allowing students to express their personal opinions and feelings, and providing opportunities for student choice. Relationship support refers to expressing positive concern, understanding and helping students learn, solving students' academic emotions and other issues (Yu, Liu, \& Zhao, 2017). In adolescence, teacher support can generally not be replaced by family support and peer support. On the one hand, teacher support can eliminate students' learning burnout by reducing their physical and mental exhaustion. Additionally, teacher support can affirm students' interests and advantages by positive feedback and improving self-efficacy to support academic and personal development. Empirical research also shows that teacher support (such as evaluation, reward and timely feedback) as environmental factors affecting students' development can trigger learning motivation and have an important impact on students' internet usage, learning input and personal development (Chai \& Gong, 2015). Given the findings, this study hypothesized that teacher support might have a direct impact on college students' math achievement as stated in Hypothesis 1: Teacher support has a significant predictive effect on college students' math achievement, and the path of influence is "teacher support $\rightarrow$ college students' math achievement."

\section{Basic Psychological Needs}

Evidence suggests that basic psychological needs include spiritual, survival or material needs $(\mathrm{Ku} \& \mathrm{Shi}, 2017)$, as well as autonomous, competence and relatedness needs. Autonomy refers to an individual perceiving that his/her behavior is determined by his/her own will and by himself/herself. Competence needs refers to the individual feeling that he/she 
is capable and has the opportunity to exercise and express his/her ability in interactions with the environment. Relatedness needs are defined as the individual feeling concern about others and cared for by others, and includes a sense of security that is subordinate to other individuals and groups (Liu \& Lin, 2013).

Self-determination theory also informs understanding the satisfaction of basic psychological needs as an important key to healthy growth and individual development. Basic psychological needs also play a mediating role in the interaction between social environment and the individual (Liu, Zhong, \& Yong, 2013). When the basic psychological needs are met, the individual will develop in a positive and healthy direction. Conversely, when the basic psychological needs are blocked, the individual will develop in a negative direction or exhibit functional disorders (Deci, Vallerand, Pelletier, \& Ryan, 1991). Research shows that the satisfaction of basic psychological needs can predict students' learning burnout and academic performance, children's online behavior and emotional development, and doctors' work engagement and job burnout (Liu, Ji, \& Wang, 2012; Liu, Shen, \& Yany, 2014; Zhen, Hu, Bao, \& Zhang, 2016). Accordingly, this study speculates that basic psychological needs have an indirect impact on the math achievement of local college students, and puts forward Hypothesis 2: Basic psychological needs play a mediating role between perceived teacher support and the students' math achievement. The influencing path is "perceived teacher support $\rightarrow$ basic psychological needs $\rightarrow$ students' math achievement."

\section{Extrinsic Motivation}

Extrinsic motivation refers to the motivation that is not caused by the activity itself, but by extrinsic stimulus or reason which has no intrinsic connection with the activity. The main characteristics of extrinsic motivation are that the individual pays much attention to extrinsic rewards, extrinsic identification, and extrinsic guidance, which are different from the main characteristics of intrinsic motivation that focuses on the individual's attention and interest in the activity (Xue, Dong, \& Zhou, 2001). According to Bloom's theory of learning motivation, external motivation is important in promoting learning and an incentive to stimulate and maintain learning behavior. Existing studies have shown that extrinsic motivation can enhance students' memory, ability to raise innovative questions, and thus improve their selfesteem, self-confidence, sense of achievement and pride, and promote learning efficiency and academic performance(Cui, 2016; Gao, 2016; Mohammed, Chowdhury, \& Shahbuddin, 2007; Wei, 2015). Bian et al.'s research further supports the mediating role of extrinsic motivation between teacher support and academic performance (Bian et al., 2012). In view of these findings, this study speculates that teacher support influences students' academic performance by influencing students' extrinsic motivation. Therefore, a third hypothesis is put forward Hypothesis 3: Teacher support 
affects students' math achievement by influencing their extrinsic motivation. The path of influence is "Teacher support $\rightarrow$ extrinsic motivation $\rightarrow$ math achievement of college students."

\section{Methodology}

\section{Participants and Procedure}

The subjects of this study were college students who had taken math courses at a local university in Guangdong Province, China. A hyperlink to a questionnaire was distributed randomly online to a total of 2,096 and 1283 questionnaires were collected. The questionnaires that missed important information and the randomness of strong invalid questionnaires were excluded. Disqualifying the invalid questionnaires 107 resulted in a total of 1176 questionnaires for analysis. The surveyed students were from mathematics, computer, electronics, chemistry, architecture, biology, economics, management, clothing and tourism programs. Of the 1176 students, 619 students were from rural areas (52.6\%), and 396 students from urban areas (33.7\%); 161 students from large and medium-sized cities (13.7\%), 396 students from small towns (33.7\%), and 619 students from countryside $(52.6 \%) ; 661$ were females $(56.2 \%)$, and 515 were males $(43.8 \%) ; 665$ were freshmen $(55.7 \%), 173$ were sophomores $(14.7 \%), 128$ were juniors (10.9\%), and 220 were senior students (18.7\%).

\section{Perceived Teacher Support Scale}

This research utilized a student perceived teacher support scale created by Yu, Liu and Zhao (2017) which measures the degree of student perceived teacher support in class. The scale includes 15 items, which are divided into three dimensions: ability support, autonomous support and relationship support. There are respectively 5,4 and 6 items in each dimension with which teachers' free choice and questioning in class are measured. For example, one item is "Teachers emphasize the importance of hard work in the learning process." In this study, Cronbach's alpha measured three dimensions with scores of $0.874,0.780$ and 0.874 respectively. Items were rated on a 5point Likert scale, ranging from 1 (Completely Disagree) to 5 (Completely Agree). With the parceling strategy, the average score of dimension synthesis score was used as the index of each variable. The higher the score, the more teacher support perceived by students. The scale was proved to have good reliability and validity with a Cronbach's alpha score of 0.93 .

\section{Basic Psychological Need Scale}

The basic psychological needs scale was compiled by Gagne (2003) and translated by Liu and Lin (2013). The scale consisted of 19 items that were divided into three dimensions: autonomous needs, competency needs and relational needs. There were respectively 6,6 and 7 items in each 
dimension. They measured the degrees of satisfaction degree among the college students' basic psychological needs in the classroom. For example, whether students can learn according to their interests and willingness in the classroom, such as "the people I like to deal with very much". Items were rated on a 5-point Likert scale ranging from 1 (Completely Disagree) to 5 (Completely Agree). Utilizing the parceling strategy, the average score of dimension synthesis score was used as the index of each variable. The higher the score, the higher the satisfaction degree of college students' basic psychological needs. The scale has been used by many researchers at home and abroad, and has good reliability and validity. The Cronbach's alpha score in this study was high at 0.91 .

\section{Extrinsic Motivation Scale}

The learning motivation scale was also employed and was translated by Chi and Xin (2006). It examined the reasons why students complete their learning tasks. There were 30 items, which were divided into two dimensions: extrinsic motivation and intrinsic motivation. This study selected 16 items from the extrinsic motivation subscale that included four sub-dimensions: dependence on others' evaluation, simple task selection, concern for interpersonal competition and pursuit of reward. There were respectively 6, 5, 4 and 2 subjects in each dimension to examine students' experience of happiness and satisfaction in the process of task completion, such as "I like relatively simple and straightforward" tasks for completing assignments. Items were rated on a 5-point Likert scale, ranging from 1 (Completely Disagree) to 5 (Completely Agree). Employing the parceling strategy again, the average score of each dimension's composite score was used as the index for each variable. The higher the score, the higher the level of students' extrinsic motivation, the more vulnerable they were to the influence of their extrinsic values. This study showed that the Cronbach's alpha score for the scale was also high at 0.85 .

\section{Academic Achievement}

In this study, the final exam was used as the statistical basis for determining the students' math achievement. The average score of the final exam assessed math analysis, higher algebra, probability theory and mathematical statistics for mathematics majors based on 20 points. Next, the score was converted into 5 points. Other majors used the average total score of high mathematics, linear algebra, probability and mathematical statistics, and then the score was converted into 5 points based on 20 points.

\section{Data Analysis}

In this study, preliminary analyses were performed in SPSS 21.00 to identify significant differences in the test scores between gender groups and between disciplines. The Harman single factor test (Zhou \& Long, 2004) was 
also used to analyze all measurement items through exploratory factor analysis. In addition, the mediation effect performed in Mplus 7.0. Structural Equation Modeling (SEM) was used to explore the relationship between variables, and to examine the mediating role of students' basic psychological needs and extrinsic motivation. Furthermore, this study used the procedural test model of mediation effect (Fang, Wen, Zhang, \& Sun, 2014; Wen \& Ye, 2014), and Mplus 7.0 to test multiple mediation models for completing the data analysis.

\section{Results}

\section{Preliminary Analyses}

Independent sample $\mathrm{T}$-test showed in Table 1 that there was no significant difference $(\mathrm{t}=0.25, p>0.05)$ in the total average score and dimensions of teacher support between genders, but there were significant differences $(t=0.04, p<0.05)$ in the total average score of teacher support and the dimension of relationship support between liberal arts students and science students. There was also no significant difference $(\mathrm{t}=0.61, p>0.05)$ in the dimension of ability support and self-support between liberal arts and science students.

Table 1. Difference in Test Scores between Gender Groups and Between

\begin{tabular}{|c|c|c|c|c|c|}
\hline \multicolumn{6}{|c|}{ Disciplines } \\
\hline $\begin{array}{l}\text { Variables } \\
(\mathrm{M}+\mathrm{SD})\end{array}$ & & $\begin{array}{l}\text { Capacity } \\
\text { support }\end{array}$ & $\begin{array}{l}\text { Independent } \\
\text { support }\end{array}$ & $\begin{array}{l}\text { Relational } \\
\text { support }\end{array}$ & $\begin{array}{l}\text { Teacher } \\
\text { support }\end{array}$ \\
\hline \multirow{2}{*}{ Gender } & $\begin{array}{l}\text { male } \\
(n=515)\end{array}$ & $3.58 \pm 0.72$ & $3.77 \pm 0.63$ & $3.75 \pm 0.67$ & $3.70 \pm 0.62$ \\
\hline & $\begin{array}{l}\text { female } \\
(\mathrm{n}=661)\end{array}$ & $3.54 \pm 0.62$ & $3.75 \pm 0.57$ & $3.69 \pm 0.65$ & $3.66 \pm 0.55$ \\
\hline \multirow{4}{*}{ Discipline } & $\mathrm{t}$ & 0.35 & 0.59 & 0.15 & 0.25 \\
\hline & $\begin{array}{l}\text { science } \\
(n=740)\end{array}$ & $3.57 \pm 0.70$ & $3.77 \pm 0.62$ & $3.77 \pm 0.66$ & $3.70 \pm 0.59$ \\
\hline & $\begin{array}{l}\text { arts } \\
(\mathrm{n}=436)\end{array}$ & $3.55 \pm 0.62$ & $3.74 \pm 0.55$ & $3.62 \pm 0.65$ & $3.63 \pm 0.55$ \\
\hline & $\mathrm{t}$ & 0.61 & 0.37 & $0.00 * *$ & $0.04 *$ \\
\hline
\end{tabular}

\section{Common Method Deviation Test}

The Harman single factor test (Zhou \& Long, 2004) analyzed all measurement items through exploratory factor analysis. It showed that the characteristic roots of 12 factors were larger than 1 , and the variance of the first factor interpretation was $16.2 \%$, far less than $40 \%$, which indicated that the common method deviation problem was not serious. The results of the multiple collinearity test show that tolerance was between 0.35 and $0.76(>$ 0.1 ), variance expansion factor (VIF) was between 1.32 and $2.87(<10)$, indicating that the problem of multiple collinearity was not serious. 


\section{Mediating Model}

In this study, Structural Equation Modeling (SEM) was used to explore the relationship between variables, and to examine the mediating role of students' basic psychological needs and extrinsic motivation. The model was constructed as follows: firstly, the direct impact of perceived teacher support on college students' math achievement was tested. Model 1 was constructed with perceived teacher support as an independent variable and math achievement as the dependent variable. Secondly, the common mediating effect of basic psychological needs and extrinsic motivation on college students' perceived teacher support and math achievement was tested, and multiple mediating Model 1 was constructed (see Figure 1).

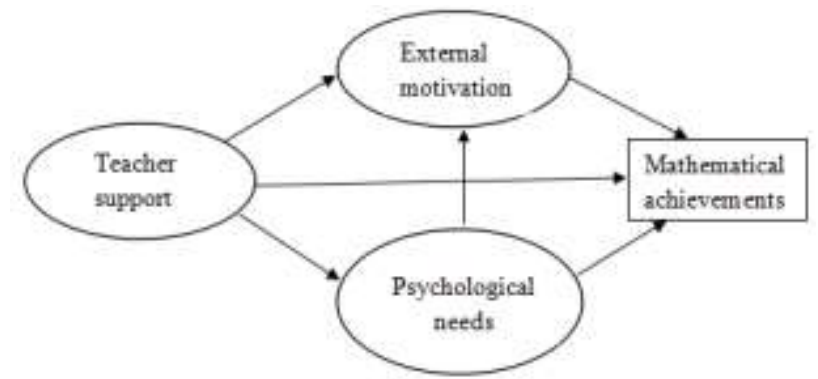

Figure 1. A multiple mediating model between teacher support and math achievement.

The absolute fitting index RMSEA was lower than the critical value of 0.08 , and the relative fitting index for Comparative Fit Index (CFI) and the Tucker-Lewis Index (TLI) were above 0.95. The final Structural Equation Modeling (SEM) is shown in Figure 2.

Indicators of multiple mediation model fitting for mathematical achievement were $\mathrm{x} 2 / \mathrm{df}=4.4$. The root-mean-square error of approximation $($ RMSEA $)=0.05$, CFI $=0.97, \mathrm{TLI}=0.96$, and the standardized root mean square residual $($ SRMR) was $=0.04$. Comprehensive indicators proved that the analytical model of this study was acceptable (Table 2).

Table 2

Index of Model Fit

\begin{tabular}{llllllll}
\hline & $\chi 2$ & $d f$ & Chi/df & RMSEA & CFI & TLI & SREMR \\
\cline { 2 - 8 } Model 1 & 0 & 2 & 0 & 0 & 1 & 1.003 & 0.002 \\
Model 2 & 177 & 40 & 4.4 & 0.054 & 0.967 & 0.955 & 0.036 \\
\hline
\end{tabular}




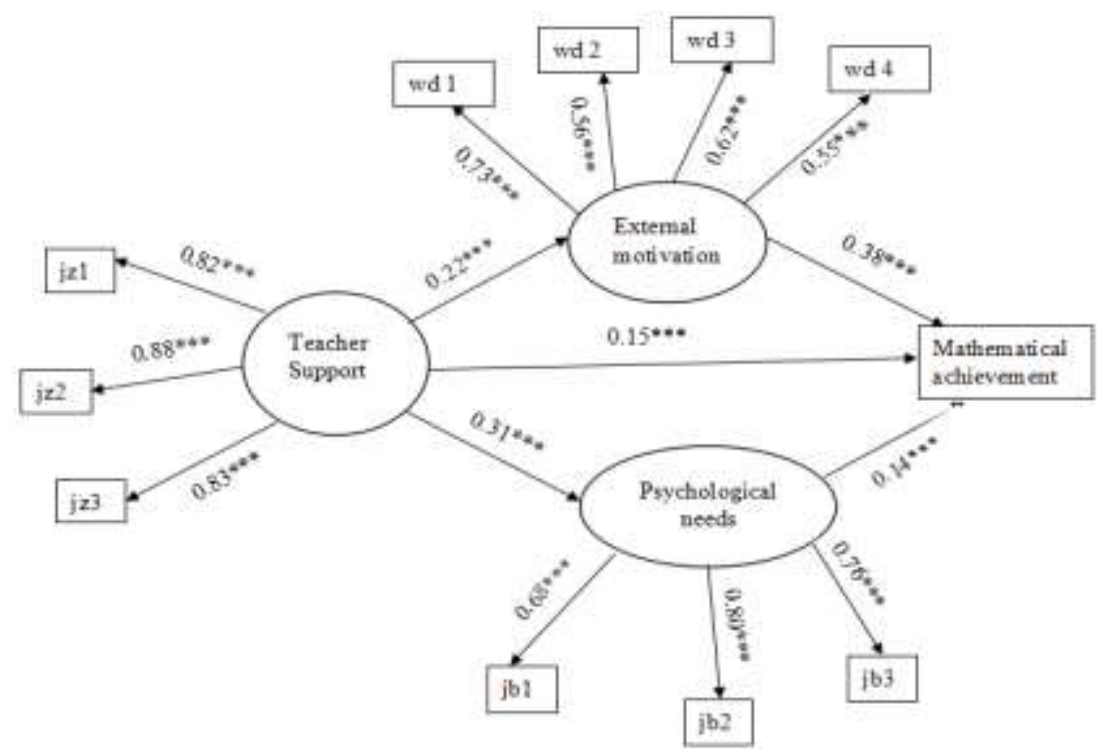

Figure 2. The final multiple mediating model between teacher support and math achievement.

\section{Testing of Multiple Mediation Models}

In this study, we used the procedural test model of mediation effect (Fang, Wen, Zhang, \& Sun, 2014; Wen \& Ye, 2014), and Mplus 7.0 to complete data analysis. The standardized regression coefficients and test results of each step are shown in Table 3. The results showed that teacher support had significant effect on students' math achievement (M1: c $=28, p$ $<.001)$; teacher support had positive effect on basic psychological needs (M2: $\mathrm{a}=31, p<.001)$; and basic psychological needs had positive effect on math achievement (M2: b = 14, $p<.001)$.

Table 3

\section{The Results of Multiple Mediation Analysis}

\begin{tabular}{lllll}
\hline & $\beta$ & $\begin{array}{c}\text { Bootstrap CI } \\
(95 \%)\end{array}$ & $\begin{array}{l}\text { Mediation effect/ } \\
\text { total effect }\end{array}$ & $\begin{array}{c}\text { Bootstrap CI } \\
(95 \%)\end{array}$ \\
\hline model & $0.151 * * *$ & {$[0.215$,} & $0151 / 0.279$ & \\
(ts-ma) & & $0.341]$ & $54 \%$ & \\
model & $0.311 * * *$ & {$[0.226,0.400]$} & $0.042 * * * / 0.279$ & {$[0.020,0.072]$} \\
(ts-bpn-ma) & $0.135 * * *$ & {$[0.060,0.204]$} & $15 \%$ & \\
model & $0.224 * * *$ & {$[0.151,0.287]$} & $0.086 * * * 0.279$ & {$[0.057,0.119]$} \\
(ts-em-ma) & $0.383 * * *$ & {$[0.305,0.449]$} & $31 \%$ & \\
$* * * 0.001$ & & & & \\
\hline
\end{tabular}

This indicated that basic psychological needs played a mediating role between teacher support and students' math achievement and was calculated by the bootstrap method. The $95 \%$ confidence interval of effect $(a b)$ is $[.02$, 
07]; the positive influence of teacher support on extrinsic motivation (M2: $\mathrm{a}=$ $22, p<.001)$, and the positive influence of extrinsic motivation on mathematics achievement $(\mathrm{M} 2: \mathrm{b}=38, p<.001)$. This showed that extrinsic motivation played a mediating role between teacher support and college students' math achievement. The 95\% confidence interval of the mediating effect (a b) calculated by bootstrap method was [.06, 12] Hypothesis 1, 2 and 3 are verified.

In addition, the indirect effects of the two mediation paths were tested (beta $=0.040, p<0.05)$, which indicated that the indirect effects of the two mediation paths $(\mathrm{xdw}=0.086 * * *, \mathrm{jb}=0.042)$ were significantly different, and the influence of the path "teacher support - extrinsic motivation - math achievement" was greater than that of the path "teacher support - basic psychological needs - math achievement" (95\% Bootstrap confidence interval). It is [0.004, 0.076].

\section{Discussion}

\section{Analysis of Gender or Discipline Differences in Teacher Support}

The results of this study shows that the total average score of teacher support and its dimensions were moderate (between 3.50 and 3.80). There was no gender difference in teacher support, and traditionally, male students were considered to have more support from teachers. The results of this study showed that gender differences were shrinking, which showed progress in math education. The perceived teacher support of college students showed discipline differences, which manifested in the total average score of teacher support and the significant disciplines differences for relationship support. Why does the perceived teacher support of college students relate to subjects? This may be because both the perceived teacher support and students' choice of subjects were related to the students' cognitive style. Students who chose science were better at acquiring knowledge from math teachers' classes, and thus perceived more support from their teachers (Chai \& Gong, 2015).

\section{The Relationship between Teacher Support and Math Achievement}

Previous studies on the relationship between perceived teacher support and college students' math achievement seldom involved local college students and the corresponding influence mechanism (Yu, Liu, \& Zhao, 2017; Zhang et al., 2018). This study included local college students as the research subjects, and explored the impact of teacher support on students' math achievement. The results showed that the teacher support had a positive effect on college students' math achievement. Basic psychological needs and extrinsic motivation played a mediating role between teacher support and college students' math achievement. The mediating role of extrinsic motivation was greater than that of basic psychological needs. This discovery validates the effect of perceived teacher support and students' math 
achievement in local colleges. In addition, it illustrated the influence mechanism on teacher support and students' math achievement, and clarified that basic psychological needs and extrinsic motivation played a mediating role between teacher support and students' math achievement (Zhang et al., 2018). Lastly, the mediating role of extrinsic motivation was found to be greater than that of basic psychological needs.

Overall, the perceived teacher support of local college students affected math achievement, but it was not enough to explain the impact mechanism. By introducing basic psychological needs and extrinsic motivations as intermediaries, this paper initiates a preliminary discussion on this issue. The research proved that teacher support affects students' math achievement through basic psychological needs and extrinsic motivation, and supports the existing theoretical and empirical research (Zhang et al., 2018). According to self-determination theory (Deci et al., 1991), when the teacher support meets the basic psychological needs of college students, the intrinsic learning motivation of college students is stimulated. Thus, encouraging college students to attain better math achievement. That is to say, if teachers in local colleges can provide a democratic, harmonious and efficient extrinsic teaching environment for students, students' basic psychological needs will therefore be met to the greatest extent and math achievement can improve.

According to learning motivation theory, the teacher support meets the extrinsic motivation of college students. That is, teachers' affirmation, positive evaluation and reward of college students' words and deeds can greatly stimulate students' self-confidence, satisfy their sense of self-esteem, stimulate their external motivation, and ultimately promote their progress in math academic achievement. This is different from the results of Zhang et al. (2018). This may be due to the lack of timely evaluation, full affirmation and specialty of local college students in primary and secondary schools, as well as the inaccuracy of their self-awareness, and the lack of clarity and clarity of their learning objectives. Learning motivation has not been strong. At university, the relaxed learning environment aggravates this phenomenon. At this time, if teachers can give strong support, it will greatly promote students' learning enthusiasm, enhance their external motivation, and then promote the improvement of math achievement.

The mediate coefficient of teacher support on math achievement through external motivation had a greater impact than that produced through the satisfaction of basic psychological needs. The results showed that although both the satisfaction of basic psychological needs and the stimulation of external motivation were mediators between perceived teacher support and college students' math achievement, there were significant differences between them, and the mediating effect of external motivation was greater than that of basic psychological needs. This may be due to the lack of selfawareness and self-awareness methods in the long-term life and education of local college students. When the outside world gives recognition to their 
words and deeds, their desire to obtain external recognition is greatly stimulated.

\section{Educational Implications}

Two insights resulted from this research. First, the score difference in gender and subject reminds us that math teachers should pay more attention to liberal arts students. Providing relationship support to liberal arts students can result in perceived respect, attention and guidance from teachers, thus stimulating learning enthusiasm, and improvement in math achievement and math quality. Second, the intermediary results of external motivation and basic psychological needs reminds math teachers that in order to implement math teaching efficiently in local colleges, teachers should attach great importance to affirming students through timely and appropriate evaluation and encouragement, promoting students' correct self-awareness and stimulating students' extrinsic motivation. However, we should not neglect to stimulate students' intrinsic motivation by satisfying their basic psychological needs. In particular, it should be emphasized that teachers should pay attention to the degree of extrinsic motivation, and avoid excessive emphasis on extrinsic motivation to arouse students' irrational worship of teachers' evaluation and reward. These precautionary steps might be necessary so that students do not ignore their correct understanding of their own ability, hindering the stimulation of intrinsic motivation that can hinder the healthy and rapid growth of college students, as the self-awareness of local college students was not very strong.

There are still some limitations and shortcomings in this study. First, the cross-sectional research design was adopted in this study. Variables except mathematics scores were measured at the same time point, so it was impossible to determine the diachronic effects of these variables. Longitudinal research methods can be used to solve this problem in future research. Second, the study did not find the mediating role of internal motivation between teacher support and math achievement, which is inconsistent with the findings from Zhang et al. (2018). In the future, we can further study the relationship between basic psychological needs and internal motivation, and find the internal mechanism that affects the relationship between them. Finally, this study's subjects were from a local university and the results do not reflect the general local university students' perceptions about the impact of teacher support on college students' math achievement. 


\section{Reference}

Bian, Y. (2012). Excessive reward transforms intrinsic motivation into extrinsic motivation Dexi's over-affirmative experiment. Mental Health Education in Primary and Secondary School, 9, 31-32.

Chai, X., \& Gong, S. (2015). Secondary school students' engagement in learning mathematics: The effects of perceived support from mathematics teachers and mathematics self-concept. Chinese Journal of Special Education, 6, 78-85.

Chi, L., \& Xin, Z. (2006). The measure of learning motivation and the relationship between it and self-efficacy of college students. Psychological development and education, 2, 64-70.

Cui J. (2016). The influence of internalization of extrinsic motivation on creative problem solving of college students. Quality Education in West China, 9, 12-13.

Deci, E. L., Vallerand, R. J., Pelletier, L. G., \& Ryan, R. M. (1991). Motivation and education: The self-determination perspective. Educational Psychologist, 26, 325-346.

Fang, J., Wen, Z., Zhang, M., \& Sun, B. (2014). Analyzing multilevel mediation using multilevel structural equation model. Psychological Science, 3, 735-741.

Gagné, M. (2003). The role of autonomy support and autonomy orientation in prosocial behavior engagement. Motivation and emotion, 27(3), 199223.

Gao, J. (2016). The relationship between extrinsic motivation and online learning engagement: A Self-determination Theory Perspective. $E$ education research, 10, 64-69.

Ku, H., \& Shi, H. (2017). Basic psychological needs: concept, structure and theoretical basis. Advances in psychology, 11, 1269-1276.

Liu, H., Ji, H., \& Wang, Y. (2012). Satisfaction of basic psychological needs as a predictor of job burnout and job engagement in physicians. Journal of Hebei University, 37, 93-99.

Liu, J., Jiang, W., Zhang, M., \& Luo S. (2016). Empirical study on the influences of college students' course interest in applied universities. Higher Education Exploration, 7, 72-77.

Liu, J. \& Lin, L. (2013). Reliability and validity of the Chinese version of the basic psychological needs Scale. Chinese Mental Health Journal, 27, 791-795.

Liu, J., Zhong, B., \& Yong, G. (2013). The application of self-determination theory among Chinese populations. Advances in Psychological Science, 21, 1803-1813.

Liu, R., Shen, C., \& Yang, Q. (2014). The effect mechanism of sensation seeking on reform school students' pathological internet use. Psychological development and education, 1, 90-104. 
Lu, G. (2010). The influence of students' perception of classroom learning environment on their approaches to learning. Fudan Education Forum, 8, 34-46.

Mohammed, S., Chowdhury, A., M., \& Shahbuddin. (2007). Self-efficacy, motivation and their relationship to academic performance of Bangladesh college students. College Quarterly, 10, 1-9.

Qiu, Z., \& Gao, J. (1999). Study on the influences of students' nonintelligence Factors from the angle of teaching. Journal of Nanjing University of Posts and Telecommunications (Social Science), 1, 43-45.

Wei, C. (2015). The influence of extrinsic motivation and cognitive style on creative scientific problem finding ability. Linfen: Shanxi Normal University.

Wen, Z., \& Ye, B. (2014). Analyses of mediating effects: the development of methods and models. Advances in Psychological Science, 22, 731-745.

Xiao, Q., Zhang, L., \& Shi, E. (2015). Statistical analysis on the influential factors of the mathematics scores of university students in different grade. Journal of Mathematics Education, 24, 53-56.

Xue, G., Dong, Q., \& Zhou, L. (2001). The relationship of intrinsic motivation and extrinsic motivation to creativity. Psychological development and education, 1, 6-11.

Ye, B., Fu, H., \& Yang, Q. (2017). Teacher's caring behavior and adolescents' internet addiction: a chain mediating model. Chinese Journal of Clinical Psychology, 6, 1168-1170.

Yu, Q., Liu J., \& Zhao Y. (2017). Study on the impact of teacher support on students' learning motivation and academic achievements. Journal of Tianjin University (Social Science), 19, 542-547.

Zhang, D., Fu, D., Liu, H., \& Liu, L. (2018). Effect of perceived teacher's autonomy support on students' achievement: the mediating role of autonomy psychological need and intrinsic motivation. Teacher Education Research, 30, 79-86.

Zhang, X., Lin, S., \& Ma, H. (2013). Statistical analysis on influence factors for mathematics achievement of college students. Journal of Southwest University for Nationalities (Natural Science Edition), 39, 21-25.

Zhen, X., Yu, C., Hu, J., Bao, Z., \& Zhang W. (2016). Basic psychological needs and adolescent internet game addiction tendency: a regulated intermediate model. Educational Measurement and Evaluation, 11: $39-45$.

Zhou, H., \& Long, L. (2004). Statistical remedies for common method biases. Advances in Psychological Science, 12,735-741. 
Authors Note: This research was supported by Guangdong province undergraduate higher education teaching reform project, by Huizhou University teaching reform project (JG2017026), and by Guangdong University Students' Innovation and Entrepreneurship Project (201810577041).

Corresponding author: Wu Hongye, School of Mathematics and Big Data, Huizhou University, 46 Yanda Street, Huicheng District, Huizhou City, Guangdong Province, 516007, China.

E-mail address: why@hzu.edu.cn.

Tel: (86)13413028749. 